\title{
Disruptive space telescope concepts, designs, and developments: OASIS and Nautilus -INVITED
}

\author{
Dae Wook Kim ${ }^{1,2, *}$, Christopher K. Walker ${ }^{2}$, Dániel Apai ${ }^{2,3}$, Tom D. Milster ${ }^{1}$, Yuzuru Takashima ${ }^{1}$, Glenn Schneider ${ }^{2}$, \\ Rongguang Liang ${ }^{1}$, Young-sik Kim ${ }^{1}$, Chuck Fellows ${ }^{2}$, Yingying Zhang ${ }^{1}$, Andras Gasper ${ }^{2}$, I. Steve Smith ${ }^{4}$, Devon Crowe ${ }^{5}$, \\ Alex Bixel ${ }^{2}$, Stefan O’Dougherty ${ }^{2}$, Siddhartha Sirsi ${ }^{2}$, Aman Chandra ${ }^{6}$, Andy Phan ${ }^{1}$, Zichan Wang ${ }^{1}$, Kira Purvin ${ }^{1}$, Henry \\ Quach $^{1}$, Marcos Esparza ${ }^{1}$, Joel Berkson ${ }^{1}$, Oliver Spires ${ }^{1}$, Heejoo Choi ${ }^{1}$, Geon Hee Kim ${ }^{7}$, and Jonathan Arenberg ${ }^{8}$ \\ ${ }^{1}$ James C. Wyant College of Optical Sciences, The University of Arizona, Tucson, AZ 85721, USA \\ ${ }^{2}$ Steward Observatory, The University of Arizona, Tucson, AZ 85721, USA \\ ${ }^{3}$ Lunar and Planetary Laboratory, The University of Arizona, Tucson, AZ 85721, USA \\ ${ }^{4}$ Southwest Research Institute, 6220 Culebra Road, San Antonio, TX 78238, USA \\ ${ }^{5}$ Raytheon Technologies, 10618 E. Rose Hill St., Tucson, AZ 85747, USA \\ ${ }^{6}$ Aerospace and Mechanical Engineering, The University of Arizona, Tucson, AZ 85721, USA \\ ${ }^{7}$ Korea Basic Science Institute, 169-148, Daejeon 34133, Republic of Korea \\ ${ }^{8}$ Northrop Grumman Aerospace Systems, Redondo Beach, CA 90278, USA
}

\begin{abstract}
Two disruptive space telescope concepts are being designed and developed at the University of Arizona; these are the 20-meter OASIS (Orbiting Astronomical Satellite for Investigating Stellar Systems) and 8.5-meter Nautilus. OASIS combines break-through inflatable aperture and adaptive optics techniques to realize the dream of a $20+$ meter class spaceborne terahertz/far-infrared telescope. In the Nautilus visible/nearinfrared telescope concept, conventional primary mirrors are replaced by an $~ 8.5$-meter MODE (Multi-order diffractive engineered) lens with 10 times lower areal density and up to 100 times lower mis-alignment sensitivity over traditional systems, enabling large-diameter optical space telescopes. The OASIS and Nautilus concepts have the potential to greatly reduce mission costs and risks compared to the current state of the art.
\end{abstract}

\section{Introduction}

The realization of a very large, space-based telescope for far-infrared/terahertz studies has long been a goal to study the origins of stars, planets, molecular clouds, and galaxies by providing an essential means of following-up on tantalizing results from recent successful missions such as Spitzer and SOFIA. The OASIS concept combines break-through technologies utilizing inflatable spherical reflectors and adaptive optics to realize a $20 \mathrm{~m}$ terahertz space telescope $[1,2]$.

In visible and near-infrared astronomy, an outstanding goal is to study the diversity of potentially Earth-like planets. Nautilus is a space telescope concept that builds on an engineered material diffractive-transmissive optical element $[3,4]$. The primary mirrors typical of current space telescopes are replaced by an $\sim 8.5$-meter MODE (multi-order diffractive engineered) lens with 10 times lower areal density, thereby enabling a lightweight structure. MODE lens telescopes also have up to 100 times relaxed alignment tolerances, compared to mirrors.

The OASIS and Nautilus concepts have the potential to greatly reduce mission costs and risks compared to current space telescope paradigms through light-weighted optical design and technology.

\section{Disruptive telescope concepts}

Two paradigm-breaking space telescopes, OASIS and Nautilus, are summarized in Table 1.

Table 1. Summary of OASIS and Nautilus [1-4]

\begin{tabular}{lll}
\hline & OASIS & Nautilus \\
\hline $\begin{array}{l}\text { Science } \\
\text { wavelength }\end{array}$ & Terahertz/ & Varible / \\
\hline $\begin{array}{l}\text { Primary } \\
\text { aperture diameter }\end{array}$ & $\sim 20$-meter & Near-infrared \\
\hline $\begin{array}{l}\text { Primary optic } \\
\text { technology }\end{array}$ & $\begin{array}{l}\text { Inflatable Hencky } \\
\text { mirror }\end{array}$ & $\begin{array}{l}\text { Replicter } \\
\text { merray option) }\end{array}$ \\
\hline $\begin{array}{l}\text { Main } \\
\text { instrument }\end{array}$ & $\begin{array}{l}\text { Heterodyne } \\
\text { receivers }\end{array}$ & $\begin{array}{l}\text { Grism-based } \\
\text { spectrometer }\end{array}$ \\
\hline
\end{tabular}

\subsection{0-meter class inflatable OASIS}

The science objectives of OASIS are met by utilizing a 20-meter inflatable aperture with heritage from the Inflatable Aperture Experiment (IAE) mission, which demonstrated in-orbit deployment of a 14-meter aperture system shown in Figure 1. OASIS will utilize a Hencky reflector geometry together with proven adaptive optics techniques to yield a wide-field-of-view inflatable Mylar mirror operating at submillimeter wavelengths (i.e., terahertz frequencies). Mylar is a polyester film made

\footnotetext{
$\overline{{ }^{*} \text { Corresponding author: letter2dwk@hotmail.com }}$
} 
from stretched polyethylene terephthalate called BoPET (Biaxially-oriented polyethylene terephthalate).

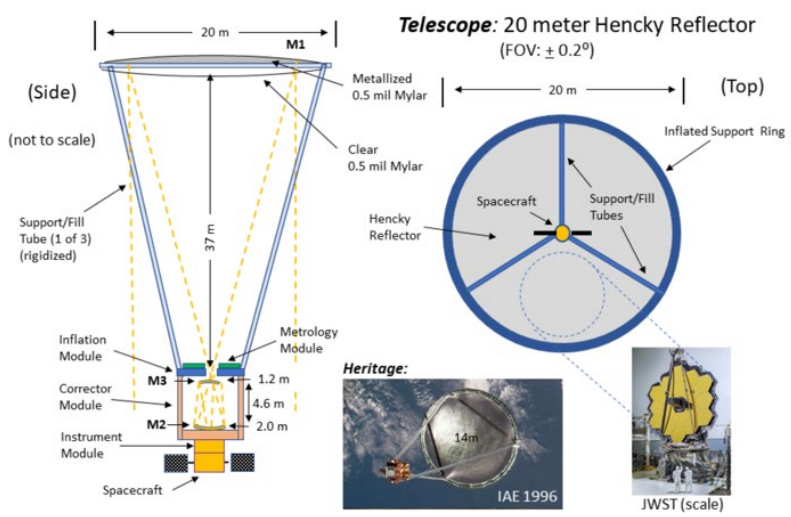

Fig. 1. Inflatable 20-meter Mylar balloon-based terahertz OASIS telescope concept design and configuration. [2]

\subsection{5-meter class replicable Nautilus}

The baseline concept for the Nautilus unit telescope in compact launch and deployed configuration is presented in Figure 2. A cross sectional view of a deployed Nautilus unit telescope is shown in Figure 2(left). The telescopes use an $8.5 \mathrm{~m}$ diameter MODE lens as the light-collecting element for exoplanet transit spectroscopy observations. Each Nautilus unit will be a stand-alone telescope equipped with two visual/near-infrared band detectors and a low-resolution spectrograph (e.g., $\mathrm{R} \sim 200$ ) optimized for the $\sim 0.45-1.6 \mu \mathrm{m}$ wavelength range.

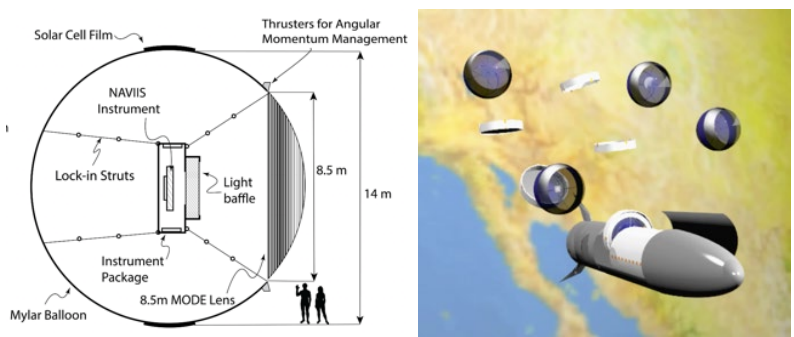

Fig. 2. A unit Nautilus telescope is launched in a compact configuration and inflated in orbit (left). Due to the compact launch configuration, multiple Nautilus units could be launched simultaneously in next-generation rocket fairings (right). [3, 4]

\section{Technology developments}

\subsection{1-meter Mylar prototype for OASIS}

The 1-meter class OASIS Mylar reflector demonstration setup has been successfully manufactured, inflated, and tested as shown in Figure 3. The Hencky surface shape and its stability as a function of time and balloon pressure has been measured using a non-null deflectometry setup.

\subsection{25-meter MODE lens camera for Nautilus}

The MODE lens has been designed considering both the diffractive and refractive nature of light and fabricated as small-scale prototypes.

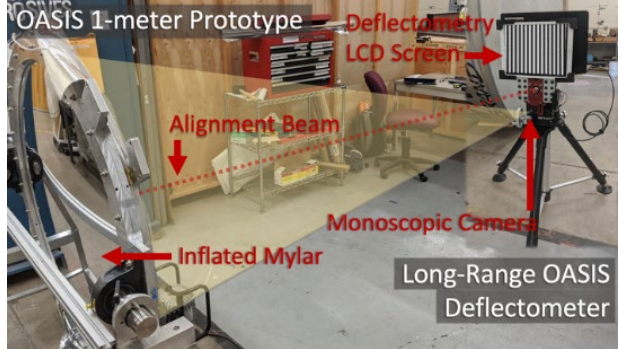

Fig. 3. 1-meter class Mylar OASIS prototype tested using the long-range deflectometry measurement setup (top-right inset).

The prototype lenses are small $(\sim 20-250 \mathrm{~mm}$ in diameter in Figure 4) and ideal for understanding the details of the fabrication trade-offs and evaluating as-manufactured optical performance. An advanced optical metrology environment has been assembled to demonstrate quantitative imaging performance.

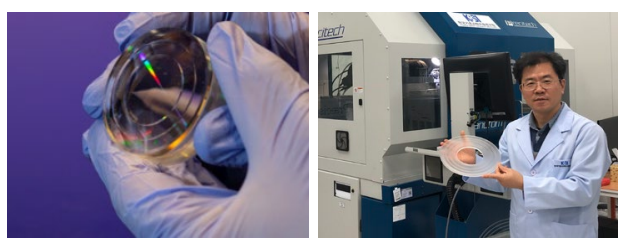

Fig. 4. MODE lens prototypes designed and fabricated with two diffractive surfaces of a front MODE surface and a single-order diffractive lens back-surface (left) [4] and a direct diamond turned $240 \mathrm{~mm}$ diameter MODE lens prototype (right).

\section{Concluding remarks}

OASIS utilizes a space-based inflatable reflector and has the potential of making the dream of space-based 20meter class telescopes a reality. Nautilus will transform astrophysics through its paradigm-breaking, scalable, and ultralight MODE lens technology.

The $>10$ fold increase in sensitivity OASIS provides will enable unprecedented access to the physical conditions of planetary bodies within our own solar system, as well as those surrounding distant stars. Large aperture visible/near-infrared wavelength spectroscopy with Nautilus will address one of the most fundamental questions in modern astrophysics by providing the first comprehensive, high-quality spectral atlas of small exoplanet atmospheres. Disruptive optics technology enables new discoveries.

\section{Acknowledgement}

This research was made possible in part by the Gordon and Betty Moore Foundation and the II-VI Foundation.

\section{References}

1. C. K. Walker, et al., IEEE MTT-S (IMS), pp. 18841887 (2017).

2. C. Walker, et al., White-Paper (2020).

3. D. Apai, et al., The Astronomical Journal, 158:83 (21pp) (2019).

4. D. Apai, et al., Proc. SPIE 11116, 1111608 (2019). 